Neurosurg Focus 19 (2):E8, 2005

\title{
Combined skull base approaches to the posterior fossa
}

\author{
Technical note
}

\author{
Nicholas C. Bambakidis, M.D., L. Fernando Gonzalez, M.D., \\ SePideh Amin-Hanjani, M.D., Vivek R. Deshmukh, M.D., Randall W. Porter, M.D., \\ Philip C. DAspit, M.D., ANd Robert F. Spetzler, M.D. \\ Division of Neurological Surgery, Barrow Neurological Institute, St. Joseph's Hospital and Medical \\ Center, Phoenix, Arizona
}

\begin{abstract}
Combined approaches to the skull base provide maximal exposure of the complex and eloquent anatomical structures contained within the posterior fossa. Common to these combined exposures are variable degrees of petrous bone removal. Understanding the advantages of each approach is critical when attempting to balance increases in operative exposure against the risk of potential complications. Despite their risks, aggressive combined exposures to the posterior fossa enable the greatest degree of visualization of the anatomy. Consequently, surgeons can approach lesions with maximal margins of safety, which cannot otherwise be realized. To minimize morbidity in all cases, the approach chosen must be applied individually, depending on the lesion and the patient's characteristics.
\end{abstract}

KEY WORDS • aneurysm • basilar artery • skull base approach • petrosectomy

The decision regarding which surgical approach to use during exposure of the posterior fossa requires careful analysis by the surgeon. The degree of exposure required obviously plays a critical role in determining the optimum route. The need to visualize the lesion and surrounding eloquent structures adequately so that resection can be undertaken safely must be weighed against the potential complications associated with each type of approach. In the axial plane, the skull base of the posterior fossa can be exposed from multiple directions, broadly classified as posterior or lateral approaches (Fig. 1A). In the sagittal plane, the options include supratentorial or infratentorial directions (Fig. 1B). In both cases, combinations of these approaches may allow the greatest degree of exposure. This maximizes the potential for safe resection of the lesion in question while limiting potential risks to the patient.

Combined approaches to the posterior fossa usually have some degree of petrosectomy as their common component. Transpetrosal approaches entail some degree of petrous (temporal) bone resection. ${ }^{6}$ Such bone removal allows maximal surgical exposure while minimizing brain retraction, and typically requires the talents of both a neurosurgeon and a neurootological skull base surgeon. Combination ap-

Abbreviations used in this paper: $\mathrm{BA}=$ basilar artery; $\mathrm{CSF}=$ cerebrospinal fluid; VA = vertebral artery. proaches in which an anterior petrosectomy and subtemporal craniotomy (Kawase approach) ${ }^{9}$ are used allow excellent exposure of the middle cranial fossa. Likewise, the posterior petrosectomy can be combined with a far-lateral or subtemporal approach (or both) to provide an unimpeded view of the entire cranial base from the foramen magnum to the clivus to the sphenoidal sinus. ${ }^{2,5,13}$ This combined approach allows exposure of the third through 12th cranial nerves, the anterolateral brainstem, and the posterior fossa vasculature from the VA to the BA apex. ${ }^{3}$

\section{GENERAL CONSIDERATIONS}

In general, we favor a supine position (Fig. 2). A shoulder roll allows rotation of the patient's head laterally, accompanied by moderate flexion of the head toward the opposite shoulder. The patient's head is positioned parallel to the floor, and the zygomatic arch is elevated. This simple and straightforward position usually enables excellent visualization of the contents of the posterior fossa. Somatosensory evoked potentials are monitored while the head is positioned. Potential changes in the waveforms may indicate compromise of the vascular system or spinal compression. Extreme rotation and flexion can cause venous congestion and engorgement, and must be avoided. 
For large patients in whom contralateral head rotation is precluded because of neck compression or shoulder elevation, a modified park bench position can be used. In this position, the dependent arm is supported by a sling and the ipsilateral shoulder is taped to minimize its profile. The use of an axillary roll to prevent brachial plexopathy is mandatory. The park bench position allows further rotation of the head and elevation of the mastoid process. This position is advantageous for approaching low-lying lesions and provides an operating angle to the anterior brainstem and foramen magnum. ${ }^{14}$ In all cases, the patient is taped securely to the operating table to allow maximal flexibility in rotation if required.

Electrophysiological monitoring is used routinely during positioning and throughout surgery. Both brainstem auditory evoked responses and somatosensory evoked potentials of the extremities are measured. Cortical activity can be assessed using electroencephalography, which confirms burst suppression during the administration of barbiturates. Facial nerve function is measured through recordings of the orbicularis oris muscle that are transduced to an audible click. The CSF is routinely drained to increase brain relaxation and to minimize the need for retraction. Postoperative lumbar drainage is associated with the reduction of CSF

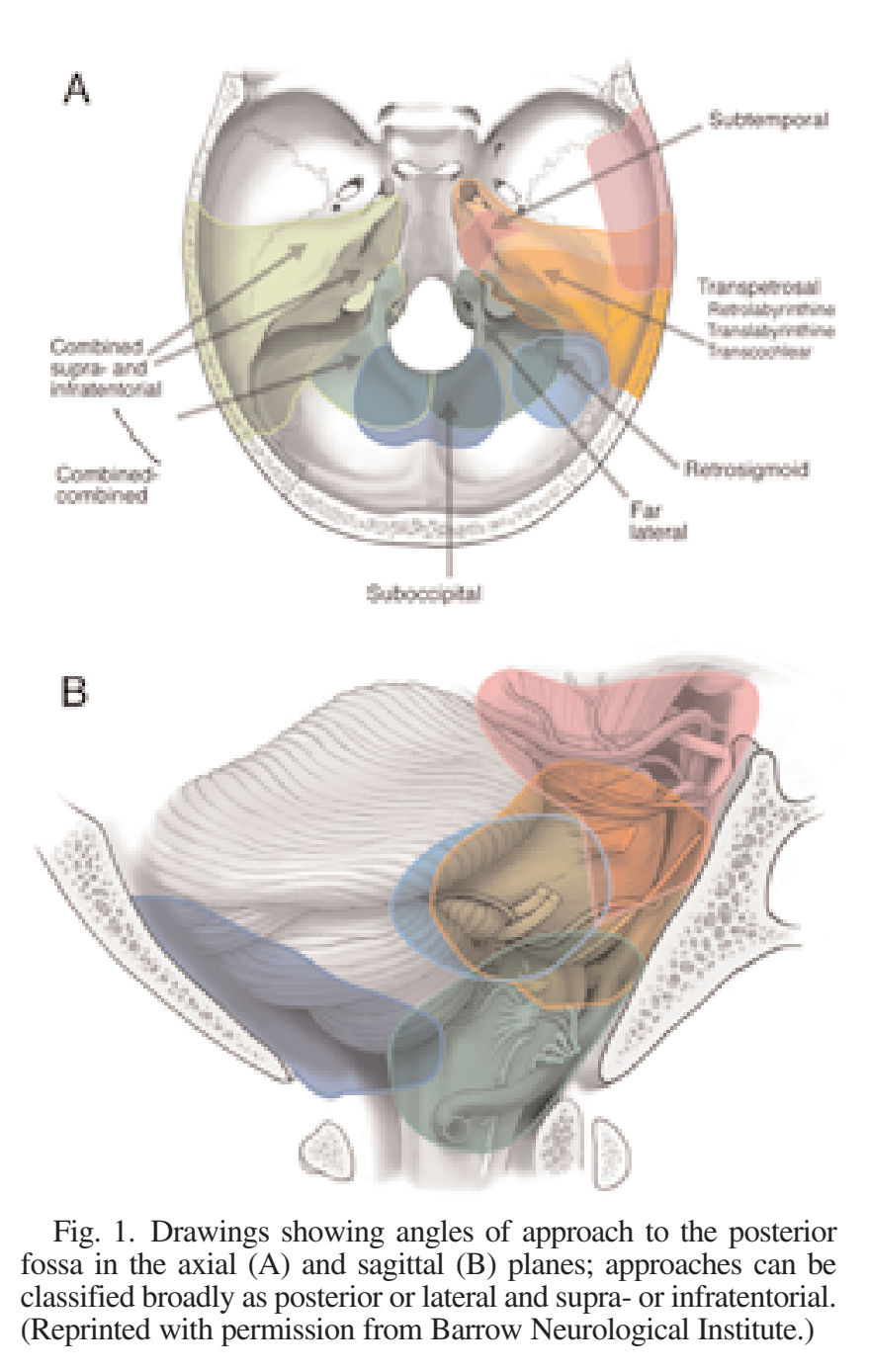

leakage-related complications, ${ }^{11}$ and typically continues for 3 to 5 days after surgery.

\section{TRANSPETROSAL APPROACHES}

Drilling the temporal bone increases exposure of the contents of the posterior and middle fossas. Progressively aggressive bone removal increases the extent of anatomical exposure obtained but likewise is accompanied by increasing rates of complications. Several variations of transpetrosal approaches have been named differently. In general, however, the posterior petrosectomies are divided into the retrolabyrinthine, translabyrinthine, and transcochlear approaches (Table 1) and are briefly described. ${ }^{5,7,8}$ The anterior transpetrosal (Kawase) approach ${ }^{9}$ is not described here. In all cases, a mastoidectomy is the initial step of bone exposure. Meticulous closure with the use of dural patch materials and fibrin glue if necessary, the use of abdominal fat grafts, and liberal lumbar spinal drainage are critical to prevent postoperative CSF leakage.

\section{Retrolabyrinthine Approach}

The retrolabyrinthine approach involves the smallest amount of bone resection. The mastoid is drilled posterior to the labyrinth and cochlea, thus preserving hearing (Fig.

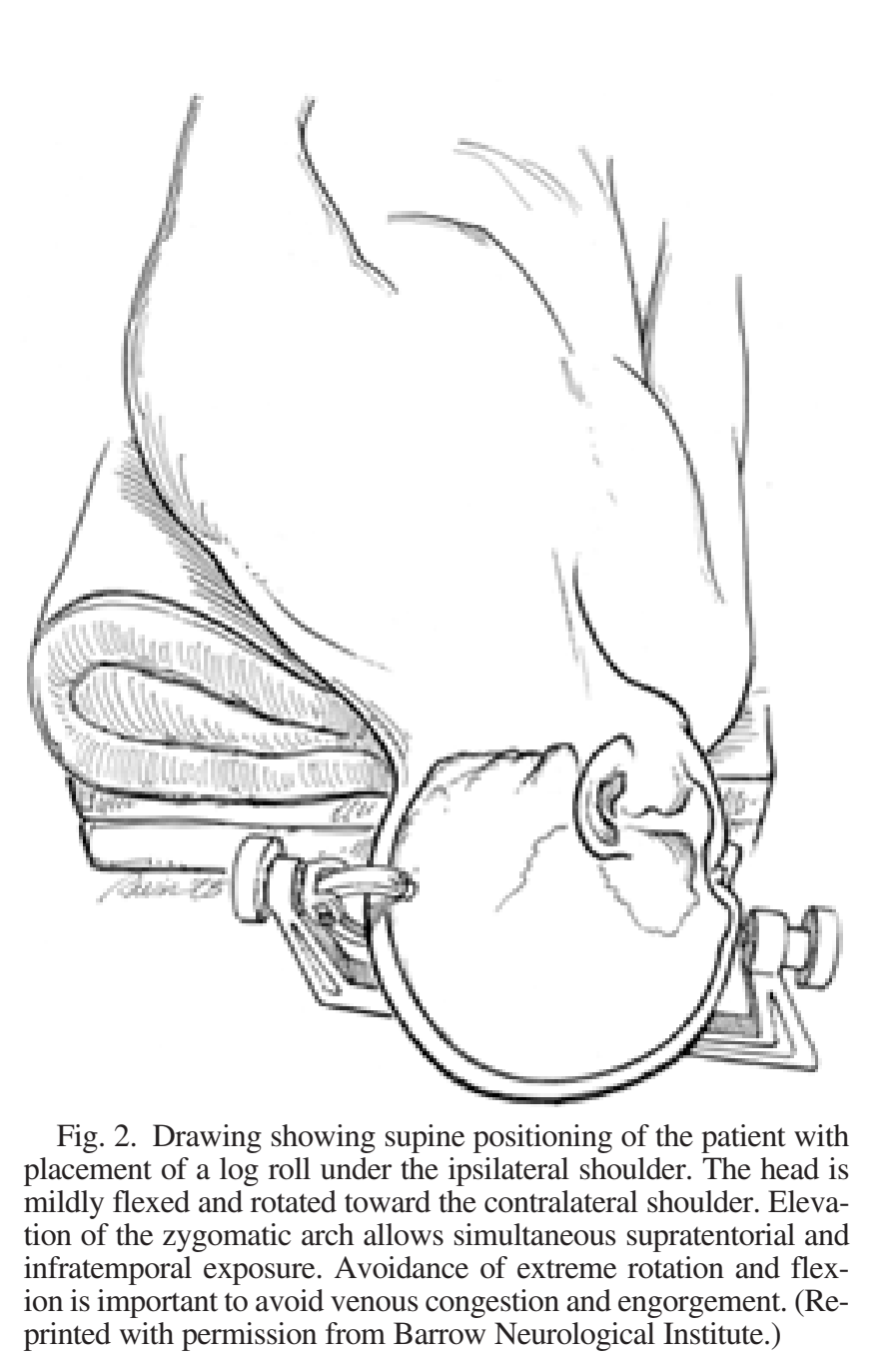


3A). When combined with a far-lateral or retrosigmoid approach, the entire sigmoid sinus is skeletonized. All surrounding dural planes are exposed with a high-powered drill. Care must be taken to avoid injury to the endolymphatic sac, which runs medial to the sigmoid sinus and inferior to the posterior semicircular canal. Damage to this structure can cause hearing loss as a result of endolymphatic fluid leakage. ${ }^{1}$

Once the dura mater is opened, the contents of the posterior fossa anterior to the sigmoid sinus can be visualized (Fig. 3B). Additional exposure may be obtained by sacrificing this sinus when the patient's venous anatomy permits such a maneuver, ${ }^{10,12}$ in combination with a traditional retrosigmoid craniotomy. In our experience, however, the slight improvement in surgical exposure seldom outweighs the added risk of venous complications and the time required to perform the bone resection. It is ideal for the treatment of vestibular neurectomies (for which the retrolabyrinthine approach was developed), but its application in other settings is limited.

\section{Translabyrinthine Approach}

Additional bone is removed in the translabyrinthine approach (Fig. 4A). Bone drilling proceeds much the same as with the retrolabyrinthine exposure, except that the entire facial nerve is completely skeletonized. The malleus and incus are removed, thus obliterating the middle ear. After a labyrinthectomy (Fig. 4B), the dura mater may be opened to provide a view of the entire subarachnoid, intracanalicular, and vertical portions of the facial nerve (Fig. 4C). Used in isolation, the translabyrinthine approach is excellent for the resection of vestibular schwannomas in patients with preoperative hearing loss. When combined with a far-lateral and the Kawase extended middle fossa approach (transtentorial and anterior transpetrosal approach), ${ }^{10}$ excellent visualization of the entire lateral brainstem is obtained.

\section{Transcochlear Approach}

Expansion of the translabyrinthine approach by removal of the external auditory canal and middle ear and further exposure of the extended facial recess define the transcochlear approach (Fig. 5). Classically described as including transposition of the facial nerve ${ }^{8}$ after division of the chorda tympani and greater superficial petrosal nerves, this approach offers the widest access to the clivus and anterior brainstem. In the transotic variation, the facial nerve is left in situ and the external auditory canal is occluded and resected. ${ }^{4}$ This variation may lessen the risk of temporary or even permanent facial paresis associated with translocation.

TABLE 1

Features of the posterior petrosectomy approaches

\begin{tabular}{llccl}
\hline \hline \multirow{4}{*}{\multicolumn{4}{c}{ Fpproach }} & \multicolumn{4}{c}{ Feature } \\
\cline { 2 - 5 } & $\begin{array}{c}\text { Hearing } \\
\text { Preservation }\end{array}$ & $\begin{array}{c}\text { Risk to } \\
\text { Facial Nerve }\end{array}$ & $\begin{array}{c}\text { Risk of } \\
\text { CSF Leakage }\end{array}$ & $\begin{array}{l}\text { Extent of } \\
\text { Exposure }\end{array}$ \\
\hline retrolabyrinthine & possible & min & low & least \\
translabyrinthine & no & min & mod & midrange \\
transcochlear & no & high & mod & most \\
\hline
\end{tabular}

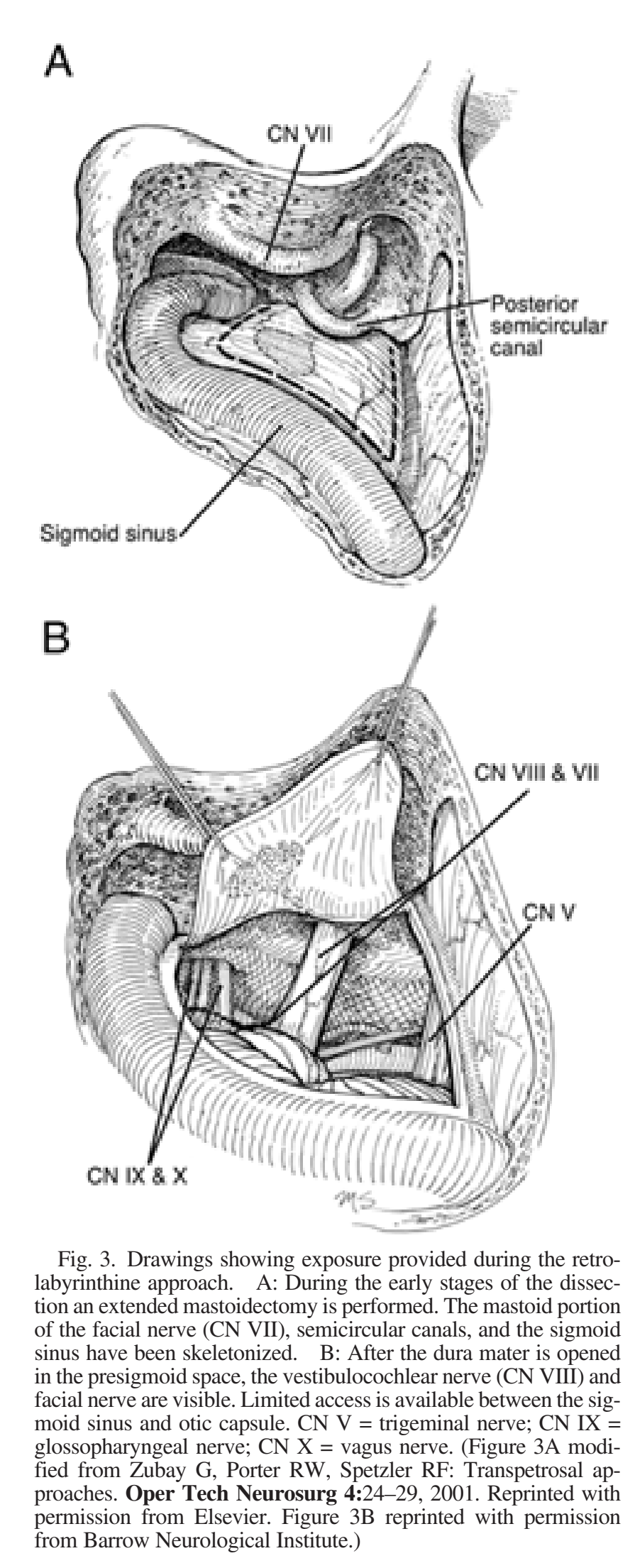




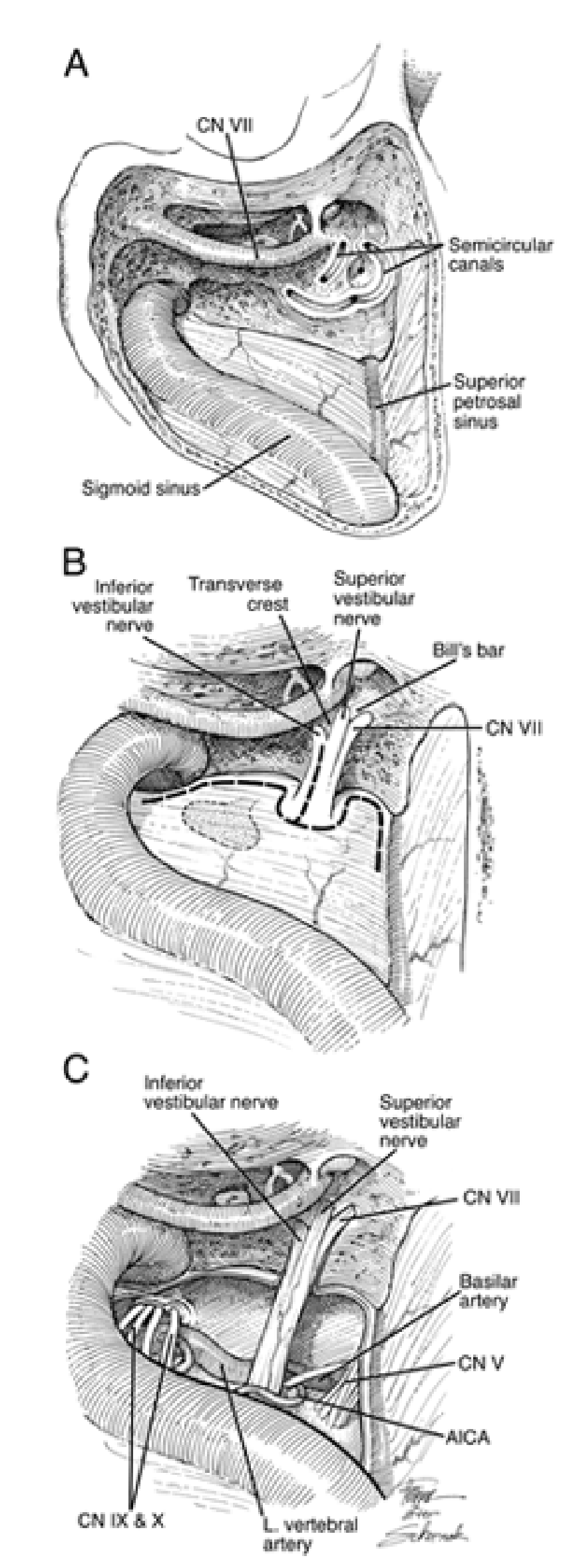

\section{COMBINED APPROACHES}

Any of the aforementioned transpetrosal approaches can be combined with standard craniotomy techniques to provide unimpeded views of the posterior fossa. The degree of exposure required depends on the location and extent of the lesion. In general, transtemporal approaches are added when lesions are large and extensive, when they involve the anterolateral brainstem or pontomedullary junction, or when they have a supratentorial component. The skin incision is easily incorporated and extended, depending on the extent of approach desired (Fig. 6A). As noted, the position of the patient's head depends on the rostrocaudal view required. The best view of the region of the foramen magnum and inferior brainstem is afforded by elevating the mastoid so that it is the highest prominence in the surgical field. Conversely, less rotation and greater elevation of the zygoma offer more direct visualization of the more rostral structures and of the tentorium.

To incorporate the far-lateral approach, we now use a paramedian incision and muscle-splitting procedure (Fig. 6B). The incision must be lateral enough to allow exposure of the mastoid yet medial enough to provide midline exposure. When the muscle-splitting procedure is used, the soft tissue must be dissected carefully to maintain the correct orientation and to avoid inadvertent injury to the VA. Constant manual palpation of the mastoid tip and lateral arch of $\mathrm{C}-1$ is mandatory. Helpful adjuncts include frameless stereotactic guidance. Subperiosteal dissection exposes the posterior fossa down to the level of the foramen magnum and to the laminae of C-1 and C-2. The incision and dissection can extend superiorly as desired, up to the level of the transverse-sigmoid sinus junction. The skin and muscle edges are reflected bilaterally with the aid of fishhooks.

Once identified, the VA is dissected from its location above the C-1 lamina to its medial entry into the intradural compartment. Most challenging at this point in the procedure is the profuse bleeding that can be encountered from the venous plexus surrounding the vessel. It is controlled by judicious bipolar coagulation and the generous use of hemostatic agents. A craniotomy may then be performed, as previously described. ${ }^{14}$ Careful dissection of the condylar vein away from the foramen magnum allows a generous bone flap to be turned. Ideally, the dissection begins at the foramen magnum laterally and extends to the midline. It continues medially up to the sigmoid sinus and jugular tubercle and courses around to the contralateral side of the foramen magnum. Additional bone may be removed as re- 


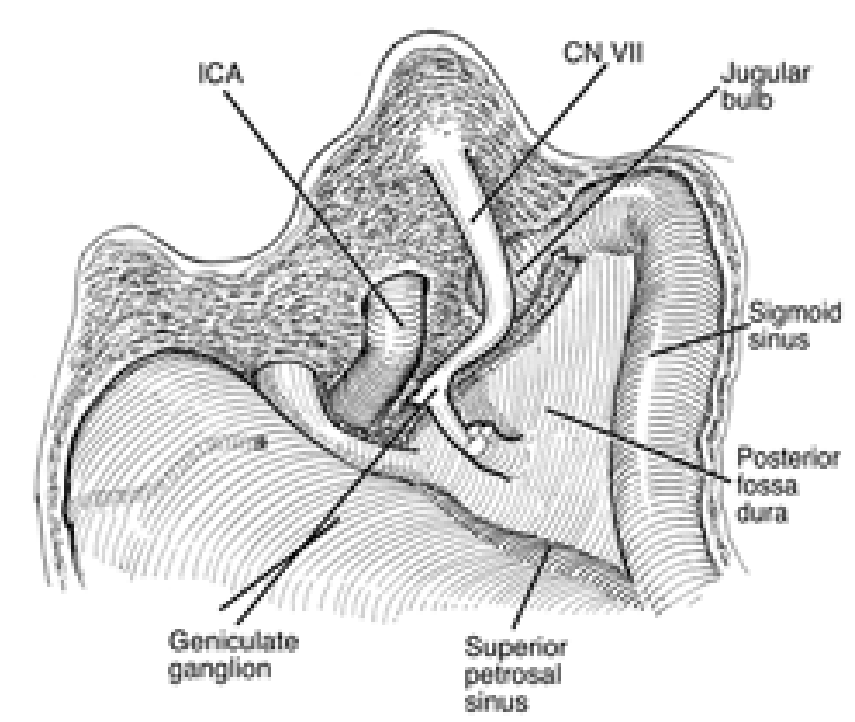

Fig. 5. Drawings showing transcochlear exposure after complete skeletonization of the facial nerve. This nerve may be transposed after the superficial petrosal and chorda tympani nerves are sectioned. Alternatively, the facial nerve may be left in situ after the external auditory canal is occluded. ICA = internal carotid artery. (Reprinted with permission from Barrow Neurological Institute.)

quired; as much as two thirds of the occipital condyle may be drilled away as needed to provide further exposure laterally. Skeletonization of the sigmoid sinus may follow, preceding additional transpetrous exposure as required to approach the lesion.
A subtemporal craniotomy is easily added by extending the skin incision superiorly and anteriorly in a curvilinear fashion. The myocutaneous flap is dissected down to the level of the zygoma and spine of Henle, and a craniotomy is turned in the usual fashion (Fig. 6C). The flap covering the transverse sinus must be turned carefully because the dura mater is often densely adherent to the bone. Crossing this junction with the drill footplate is seldom advisable. Instead, the sinus is skeletonized carefully with the cutting or diamond burr. Incorporation of both the far-lateral approach and the subtemporal craniotomy with various degrees of petrosectomy allows the entire posterior temporal, retrosigmoid, and lateral suboccipital regions to be incorporated.

After the bone is removed, the dura mater can be opened in many different ways (Fig. 7). The infratentorial opening usually begins in the midline inferiorly and proceeds superiorly and laterally to the transverse-sigmoid junction. A separate dural incision may be made linearly and anteriorly along the sigmoidal sinus to allow simultaneous visualization of the presigmoid space with minimal cerebellar retraction (Fig. 7A). Simultaneous opening of the retrosigmoid dura extended inferiorly below the foramen magnum allows simultaneous visualization of the structures of the inferior posterior fossa and brainstem, as in a standard farlateral approach (Fig. 7B). The greatest degree of visualization is obtained by dividing the sigmoid sinus as well (Fig. 7C). Nevertheless, this maneuver is only possible if the patient's venous anatomy allows drainage of the ipsilateral vein of Labbé into the contralateral transverse and sigmoid sinuses (Fig. 8). Subtemporal dural openings are started over the temporal lobe anteriorly and extended posteriorly to the superior petrosal sinuses. Care must be taken to avoid injury to the vein of Labbé or to associated veins attached

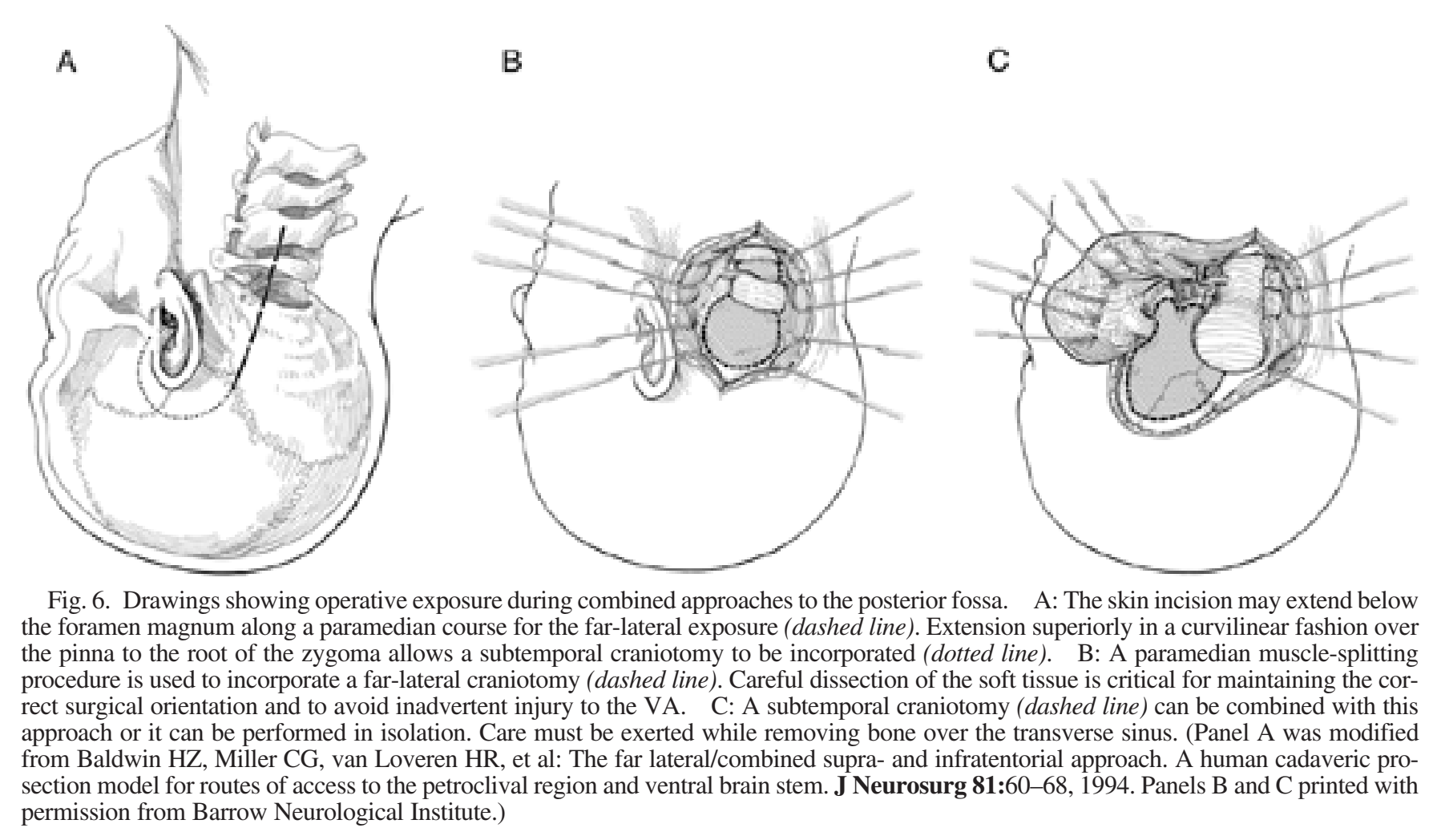



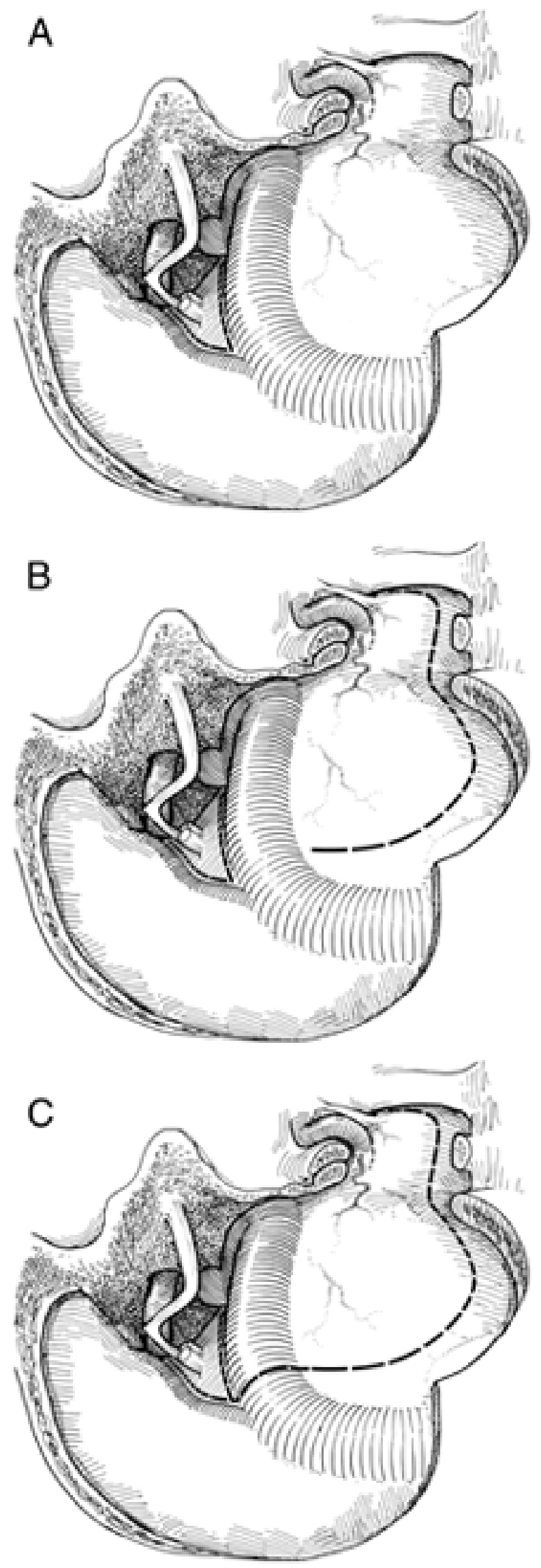

to the temporal dura mater or tentorium. An additional opening is made anterior to the sigmoid sinus, up to the sinodural angle (Citelli sinodural angle). The superior petrosal sinus is then divided, and the opening is extended along the tentorium (Fig. 9). Care must be taken to avoid damage to the fourth cranial nerve as it courses along the tentorial edge, lying medial and inferior to the temporal lobe, before its insertion at the medial tentorial edge. If necessary and if the venous anatomy is favorable, the sigmoid sinus may be sacrificed by extending the dural incision across it. Such a maneuver allows an unimpeded view of the contents of the posterior and inferior middle fossa from the inferior brainstem to the upper clivus. The anterosuperior brainstem, cavernous sinus, and Meckel cave are visualized clearly. Nevertheless, the venous structures often can be left intact. Sequential movement of the operating microscope and judicious use of the retractor allows adequate visualization while minimizing the risk of venous injury or infarction (Fig. 10). The exact approach and degree of exposure must be tailored to the lesion and the needs of each patient to allow adequate surgical treatment associated with an acceptable rate of morbidity.

\section{ILLUSTRATIVE CASE}

History and Examination. This 51-year-old man with a history of hypertension and heavy tobacco use experienced progressively worsening headaches over several months. The headaches were accompanied by the onset of diplopia, which was worse with right gaze. His physical examination revealed a right-sided sixth cranial nerve palsy. There were no other abnormal findings. Neuroimaging studies revealed the presence of a large midbasilar aneurysm (Fig. 11, Video 1).

Video 1. A rotating view of the preoperative three-dimensional angiogram showing a large wide-necked midbasilar aneurysm in the patient presented in the Illustrative Case. (Click here to view with Windows Media Player, on here to view with RealPlayer.)

Operation. The patient underwent a right combined transcochlear presigmoid/retrosigmoid approach for direct clip occlusion of the aneurysm, which was performed with the assistance of intraoperative hypothermic cardiac standstill (Fig. 12, Video 2). During the dissection, the facial nerve was transposed after the greater superficial petrosal nerve was sectioned. Patency of the BA and obliteration of the aneurysm were confirmed with postoperative angiography (Video 3).

Fig. 7. Drawings depicting how, after the transverse and sigmoid sinuses have been skeletonized, the dura mater may be opened in several ways. A: Opening (dashed line) along the presigmoid dura mater allows visualization of the anatomy with minimal cerebellar retraction. B: Simultaneous cuts in the retrosigmoid dura (longer dashed line) may be extended inferiorly to below the foramen magnum. C: Alternatively, the sigmoid sinus may be divided (extended dashed line). (Modified from Baldwin HZ, Miller CG, van Loveren HR, et al: The far lateral/combined supra- and infratentorial approach. A human cadaveric prosection model for routes of access to the petroclival region and ventral brain stem. J Neurosurg 81: $60-68,1994$. 


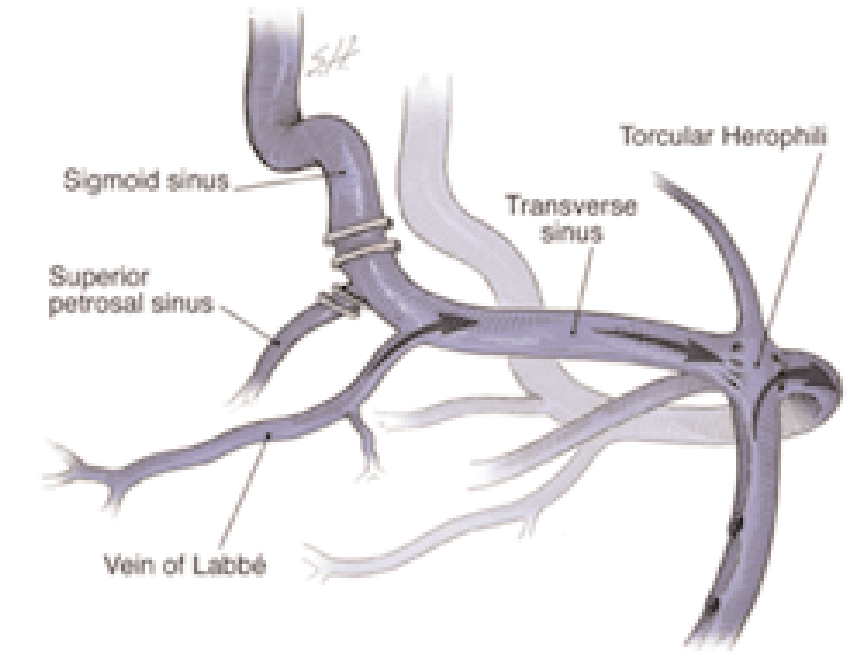

Fig. 8. Drawing showing the surgical view of the venous sinuses of the posterior fossa. Before the sigmoid sinus is ligated, patency of the contralateral jugular venous system must be confirmed. After the superior petrosal and sigmoid sinuses have been divided, blood is diverted through the torcular herophili down the transverse-sigmoid sinus to the opposite jugular vein. (Reprinted with permission from Barrow Neurological Institute.)

Video 2. Intraoperative video obtained in the patient presented in the Illustrative Case. The initial view shows the opening of the presigmoid dura mater below the transposed facial nerve after transcochlear exposure. Next is shown the initial

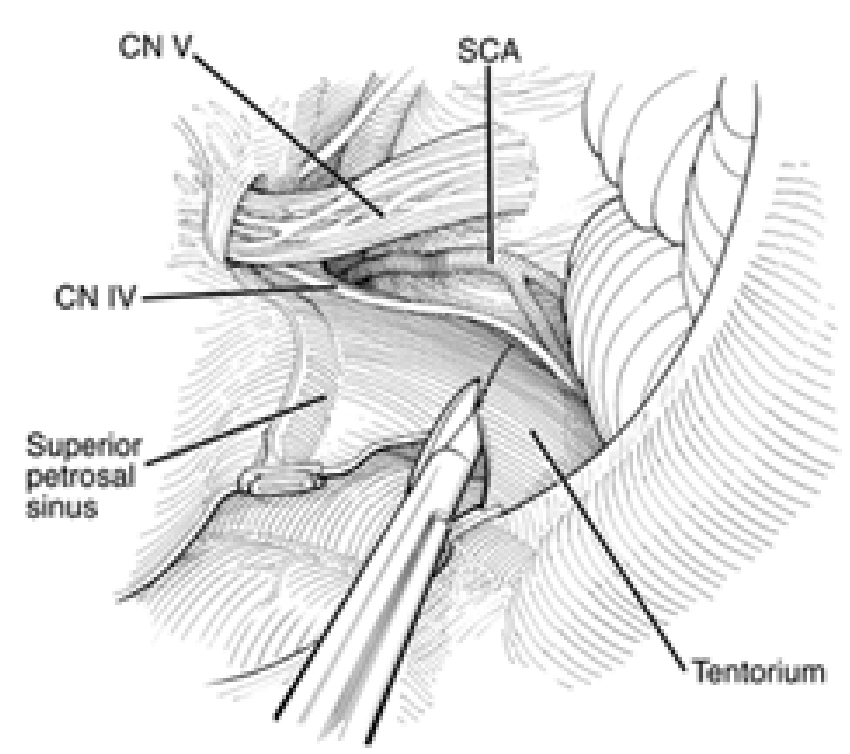

Fig. 9. Drawing showing the extent of exposure afforded by a combined transcochlear far-lateral and subtemporal exposure. The superior petrosal sinus has been divided, and further exposure is afforded by division of the tentorium. During this maneuver, care must be taken to avoid damage to the trochlear nerve (CN IV), which courses along the tentorial border. SCA = superior cerebellar artery. (Reprinted with permission from Barrow Neurological Institute.)

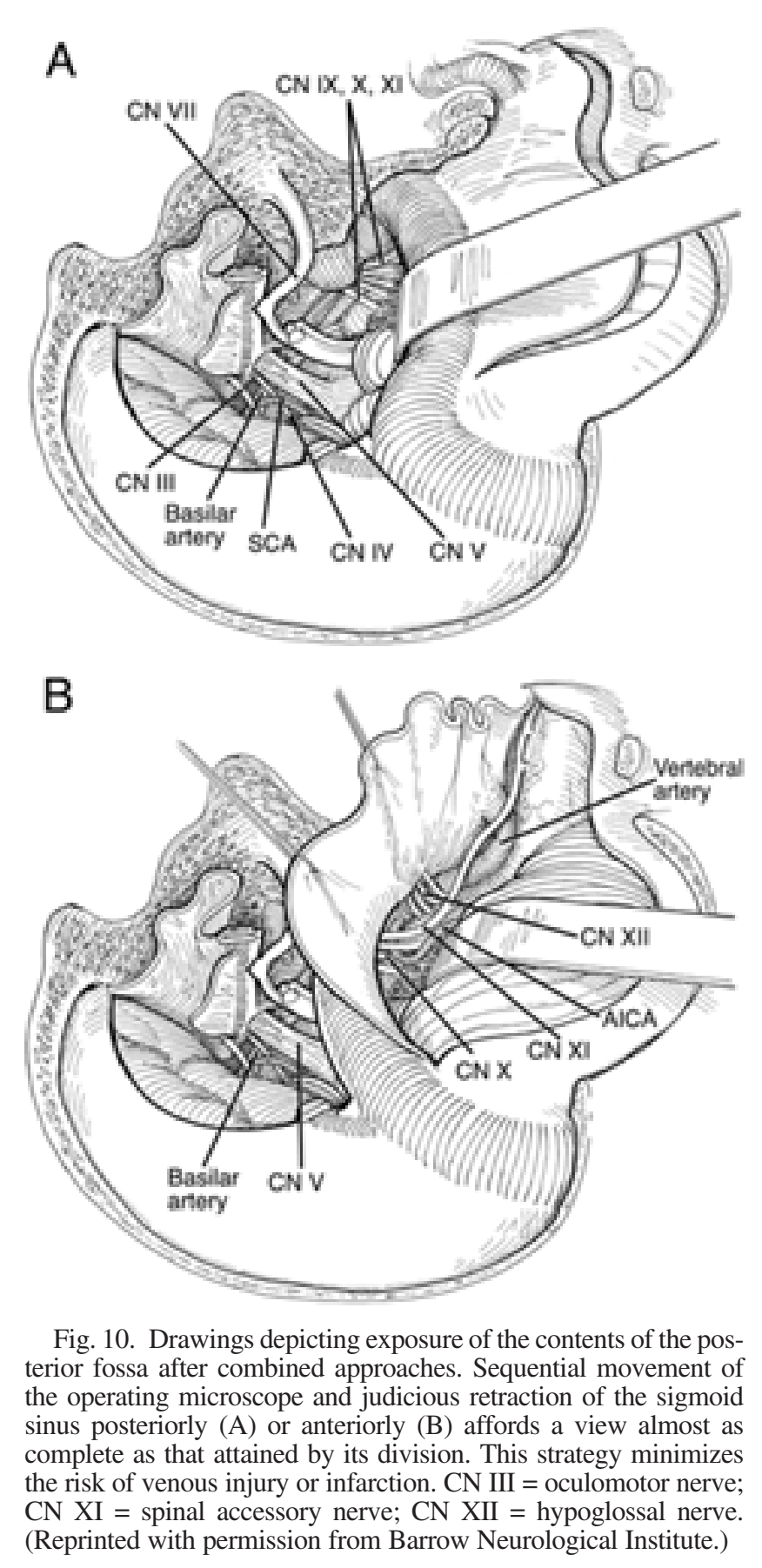

view of the aneurysm dome before cardiac standstill is induced. Dissection proceeds above the dome until the proximal BA comes into view, as hypothermic circulatory arrest is initiated. Next, dissection proceeds superiorly around the fifth cranial nerve to the distal BA, which is carefully dissected away from the anterior surface of the brainstem. Identification and preservation of perforating vessels on either side of the basilar trunk precede definitive clip placement. The aneurysm dome collapses after the blood is diverted from the venous circulation into the bypass pump. Clip placement demands meticulous attention to preserve the perforating vessels. (Click here to view with Windows Media Player, or here to view with RealPlayer.) 


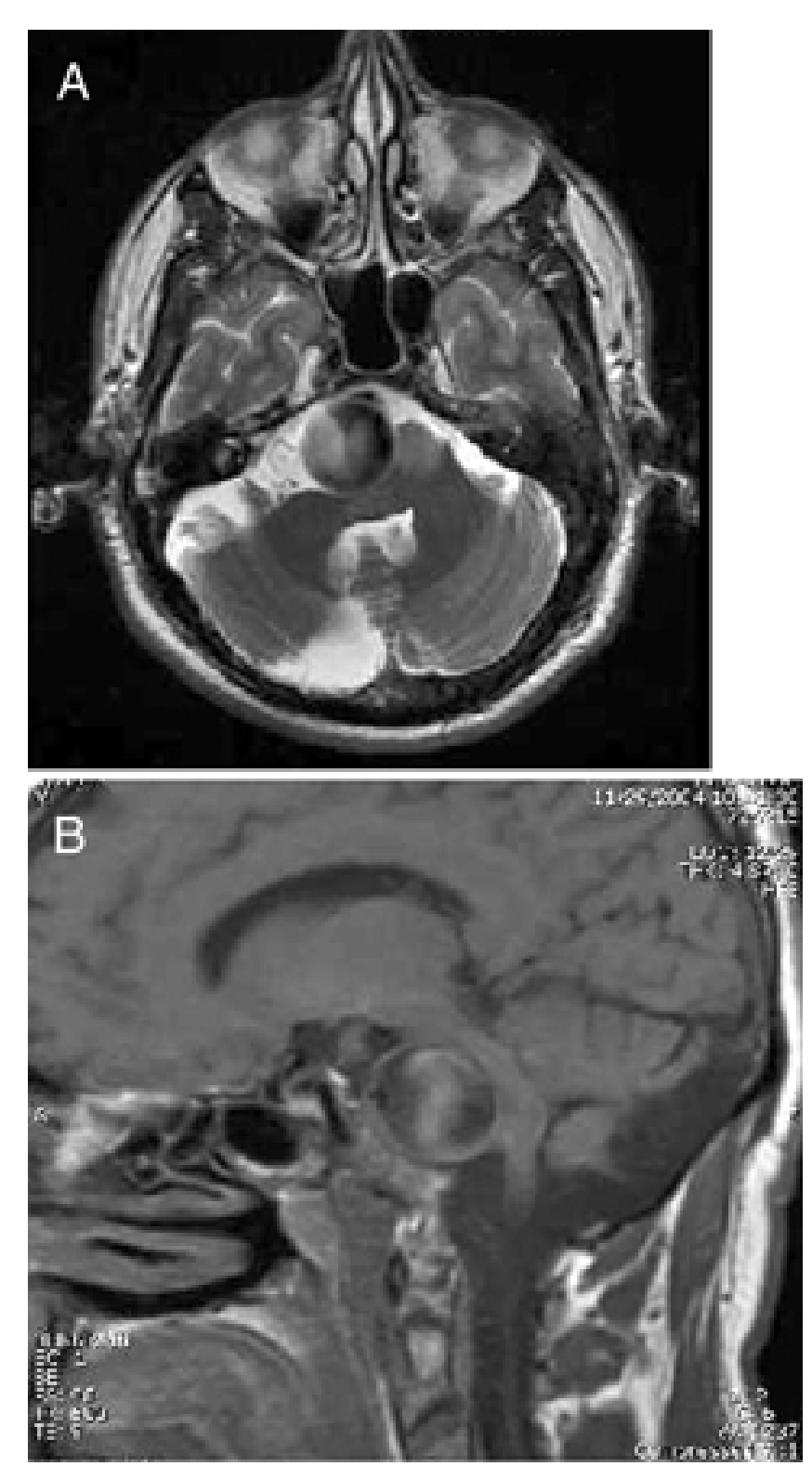

Fig. 11. Preoperative axial $\mathrm{T}_{2}$-weighted (A) and sagittal $\mathrm{T}_{1^{-}}$ weighted (B) MR images demonstrating a giant midbasilar aneurysm associated with brainstem compression.

Video 3. A postoperative three-dimensional angiogram shows marked reduction in the size of the aneurysm and confirms patency of the BA. (Click here to view with Windows Media Player, or here to view with RealPlayer.)

Postoperative Course. Postoperatively, the patient had a House Grade V facial nerve paresis, continued right gaze paresis, and a mild left hemiparesis along with right-sided hearing loss. Despite aggressive postoperative lumbar drainage, he experienced persistent CSF leakage that necessitated revision of his wound and placement of a permanent CSF shunt. Four months after surgery, his facial weakness had improved to House Grade III and his hemiparesis had resolved.

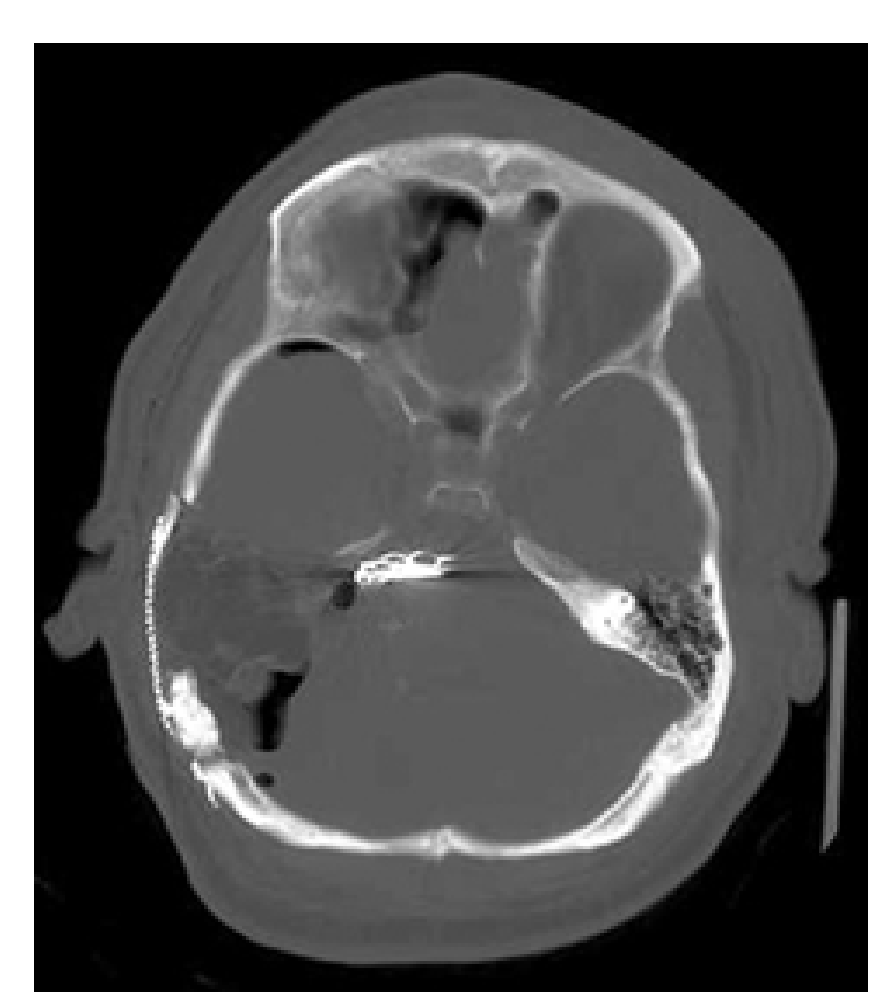

Fig. 12. Postoperative axial computerized tomography scan demonstrating the extent of bone removal achieved during the transcochlear approach. The aneurysm clips are positioned anterior to the brainstem.

\section{Acknowledgments}

We thank Mark Schornak, Spencer Phippen, Deborah Ravin, and Stephen Harrison for their anatomical drawings.

\section{References}

1. Ammirati M, Spallone A, Feghali J, et al: The endolymphatic sac: microsurgical topographic anatomy. Neurosurgery 36: 416-419, 1995

2. Baldwin HZ, Miller CG, van Loveren HR, et al: The far lateral/combined supra- and infratentorial approach. A human cadaveric prosection model for routes of access to the petroclival region and ventral brain stem. J Neurosurg 81:60-68, 1994

3. Baldwin HZ, Spetzler RF, Daspit CP: Extended transpetrosal approaches to the craniovertebral junction, in Dickman CA, Spetzler RF, Sonntag VKS (eds): Surgery of the Craniovertebral Junction. New York: Thieme, 1997, pp 491-506

4. Fisch U: Infratemporal fossa approach to tumors of the temporal bone and base of the skull. J Laryngol Otol 92:949-967, 1978

5. Hitselberger WE, House WF: A combined approach to the cerebellopontine angle. A suboccipital-petrosal approach. Arch Otolaryngol 84:267-285, 1966

6. Horgan MA, Anderson GJ, Kellogg JX, et al: Classification and quantification of the petrosal approach to the petroclival region. J Neurosurg 93:108-112, 2000

7. House WF: Translabyrinthine approach, in House WF, Luetje CM (eds): Acoustic Tumors. Baltimore: University Park Press, 1979, Vol 2, pp 43-87

8. House WF, Hitselberger WE: The transcochlear approach to the skull base. Arch Otolaryngol 102:334-342, 1976

9. Kawase T, Shiobara R, Toya S: Anterior transpetrosal-transten- 


\section{Combined skull base approaches to the posterior fossa}

torial approach for sphenopetroclival meningiomas: surgical method and results in 10 patients. Neurosurgery 28:869-876, 1991

10. Kawase T, Toya S, Shiobara R, et al: Transpetrosal approach for aneurysms of the lower basilar artery. J Neurosurg 63: 857-861, 1985

11. Laing RJ, Smielewski P, Czosnyka M, et al: A study of perioperative lumbar cerebrospinal fluid pressure in patient undergoing acoustic neuroma surgery. Skull Base 10:179-186, 2000

12. Maceri DR, Giannotta SL: The combined retrolabyrinthine/retrosigmoid approach. Oper Tech Neurosurg 2:48-51, 1999

13. Spetzler RF, Daspit CP, Pappas CT: The combined supra- and infratentorial approach for lesions of the petrous and clival re- gions: experience with 46 cases. J Neurosurg 76:588-599, 1992

14. Thompson BG, Spetzler RF: Far lateral suboccipital approach to the craniovertebral junction, in Dickman CA, Spetzler RF, Sonntag VKS (eds): Surgery of the Craniovertebral Junction. New York: Thieme, 1997, pp 467-481

Manuscript received June 20, 2005

Accepted in final form July 15, 2005.

Address reprint requests to: Robert F. Spetzler, M.D., Neuroscience Publications, Barrow Neurological Institute, 350 West Thomas Road, Phoenix, Arizona 85013. email: neuropub@chw.edu. 